\title{
Recognition in review
}

\author{
Each year, hundreds of scientists dedicate their time and expertise to help us assess the manuscripts that we send \\ out for review. For those papers that make it through to publication, we will now be thanking them publicly.
}

$\mathrm{N}$ o scientific journal can function without the trust of the wider research community that it serves. This trust is manifested in many ways, but none is quite as important as the peer-review process that underpins the quality and robustness of scientific claims being made in the scholarly literature.

Historically, most journals have tended to review the papers they receive confidentially, using the 'single-blind' approach: the reviewers' comments and feedback are relayed to the authors, but their identities are not. At all Nature research journals, the peer-review process is managed exclusively by full-time, professional editors, and one of their most important jobs is to ensure its quality and integrity. In practice, this means that special emphasis is placed on the selection of referees: their areas of expertise, scientific experience and professionalism are all factors that play a role, alongside other aspects such as geographical and gender diversity. Ultimately, we are after the best scientific advice we can get, wherever it may come from - our referee pool is therefore not a club.

Peer review is a much maligned process - typical complaints are that it can be slow, inefficient and arbitrary - but on the whole we find that it works: our authors value receiving detailed and high-quality reports on their manuscripts, and while it is never pleasant being on the receiving end of any critique, it inevitably shines light on the relative strengths and weaknesses of ones work.

Indeed, Nature research editors can recall many situations in which the feedback from reviewers was crucial for strengthening a particular claim, or pointing out wider implications that the authors themselves hadn't fully considered. Perhaps more importantly, they can also recall countless examples demonstrating the integrity and professionalism of the scientists they work with - and hence the integrity of the process itself. We find that there is a discrepancy between the perception and the reality of the peer-review procedure that we manage: the vast majority of referees do a fantastic job, and this deserves to be recognized.

It is therefore surprising and, yes, a little bit embarrassing that we haven't done more to acknowledge how much we value the critical input from referees in the past. Our deference to confidentiality is the main explanation for this. However, we are now confident that the judicious introduction of transparency enhances trust, and it is for this reason that, along with nine other Nature research journals, we now give all reviewers of our published papers the option to be formally and publicly recognized for their contribution to the peer-review procedure.

In fact, a referee recognition trial was originally rolled out at Nature in March 2016. Over the last three years, over 3,300 Nature referees across the natural sciences have chosen to be publicly recognized and $\sim 80 \%$ of Nature papers have at least one referee named. We expect this trend to hold at Nature Physics as well.

Public recognition of our reviewers is only a small step towards transparency and, unlike our sister journal Nature Communications, we don't yet feel ready to implement transparent peer review and publish reviewers' reports alongside our papers - that is a conversation for the (near) future. Nevertheless, to our reviewers we say: thank you. We value and recognize your essential contribution to the scientific endeavour as a whole.

Published online: 1 March 2019

https://doi.org/10.1038/s41567-019-0473-0

\section{March of physics}

In praise of the March meeting of the American Physical Society.

$\mathrm{N}$ ext week, over 11,000 physicists will gather in Boston for the annual American Physical Society (APS) March meeting. Be they first-time attendee students or seasoned old timers, it doubtless represents a highlight in the physics calendar.

The meeting is inextricably associated with condensed-matter physics, but it now represents 28 APS units and committees spanning areas as wide ranging as atomic, molecular and optical physics, biological physics, and quantum information. How did the conference get so big? While commercial factors certainly play a role, the deeper historical reasons are tied to the evolution of the APS as an organization and indeed the post-war rise of solid-state physics and its expansionary descendant, condensed-matter physics (J. D. Martin, Physics Today 72, 30; 2019) - a topic discussed in detail in Joseph Martin's recent book Solid State Insurrection: How the Science of Substance Made American Physics Matter.

Most years, the meeting remains memorable for the buzz surrounding a particular topic that has taken the community by storm. Most famously, the 1987 meeting in New York has come to be remembered as the Woodstock of physics, such was the excitement generated by the recent discovery of high-temperature superconductivity in copper oxides.

Other vivid memories of overflowing rooms packed with excited researchers include 2007 in Denver (the topic creating waves that year was graphene), 2010 in
Portland (topological insulators) and last year in Los Angeles (bilayer graphene, slightly twisted, turns out to be superconducting). There is also that time in Las Vegas in 1986, when attendees were so taken in by the physics that it prompted a particularly downbeat local newspaper headline: 'Physicists in town, lowest casino take ever.' The rumour is that no Las Vegas hotel has put in a bid for the March meeting since.

What will the excitement be about this year? We couldn't possibly say. What we do know is that it will be fascinating and fun in equal measure. Enjoy.

Published online: 1 March 2019 https://doi.org/10.1038/s41567-019-0474-z 